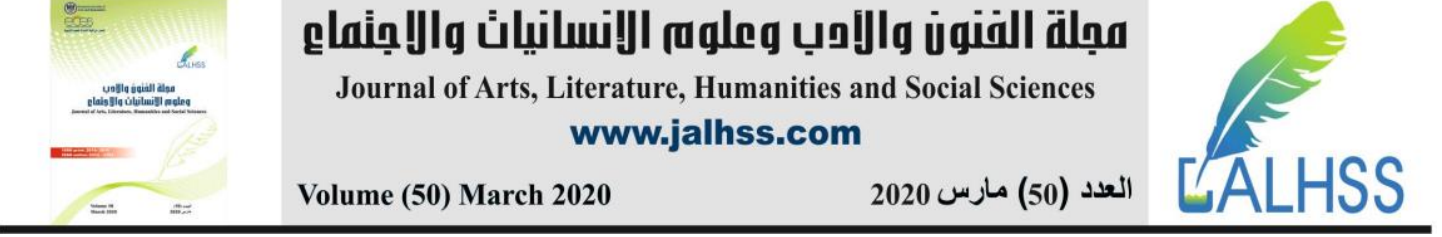

\title{
التوقيع الرقمي: تطور تكنولوجي لحجية قاتونية
}

الأستاذ الاكتور ياسر وجيه صلاح

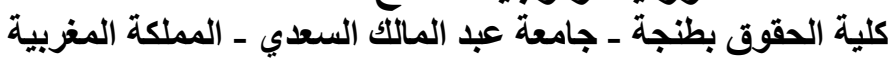

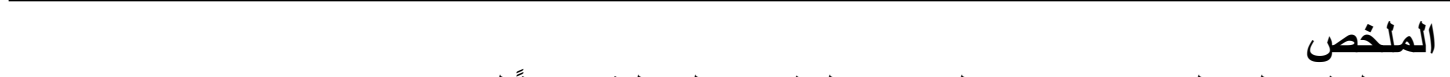
ساهم التطور التكنولوجي في تحسين العديد من القطاعات المختلفة، نتيجةً لما تم تقديمه من معدات وتقنيات جديدة تعمل على توفير وقت وجه الإنسان، ومن أبرز القطاعات التي ازدهرت مع التطور التكنولوجي القطاع الاقتصادي باستثمار اته و تعاقداته.

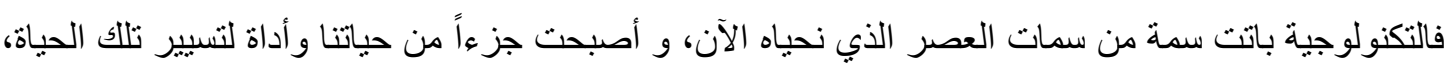

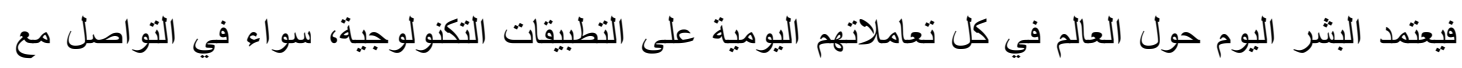
الآخرين أو إنجاز أعمالهم، أو حتى الترفيه. و نتج عن التطور التكنولوجي ظهور وسائل جديدة للتعاقد، ترتب عنها تغير في مجموعة من الدفاهيم القانونية حتى تستجيب لخصوصيات العصر الحديث ، هذه التحولات شملت على الخصوص الإثبات و التوقيع، من خلال هذا البحت سنقوم بتسليط الضوء على مختلف التعاريف القانونية و الفقهية لمفهوم التوقيع الإلكتروني ثم نبين موقع التوقيع الإلكتروني بين الهوية و الإر ادة ومكانته كحجية قانونية ملزمة. الكلمات المفتاحية: القانون، التكنولوجيا، التوقيع الرقمي، الاقتصاد الرقمي، التطور، الحجية و الإثبات. 
Lims

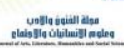

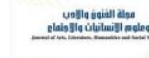

Journal of Arts, Literature, Humanities and Social Sciences

www.jalhss.com

\title{
Digital Signature: A Technological Evolution of Legal Authenticity
}

\author{
Yasser Wajih Salah \\ Faculty of Law of Tangiers - University of Abdelmalek Saadi - Kingdom of Morocco
}

\begin{abstract}
Technological development has contributed to the improvement of many different sectors, as a result of the new equipment and technologies that have been presented that save human time and effort. Among the most prominent sectors that have flourished with technological development is the economic sector with its investments and contracts. Technology has become a feature of the era in which we live now, and it has become a part of our lives and a tool for managing that life. Today, people around the world depend in all their daily dealings on technological applications, whether in communicating with others or completing their work, or even entertainment, The technological development resulted in the emergence of new means of contracting, which resulted in a change in a set of legal concepts in order to respond to the peculiarities of the modern era. These transformations included, in particular, proof and signature.

Through this research, we will shed light on the various legal and jurisprudential definitions of the concept of electronic signature, and then show the location of the electronic signature between identity and will and its status as a binding legal authority.
\end{abstract}

Keywords: Law, technology, digital signature digital, economy, Development, Authentic and Evidence. 


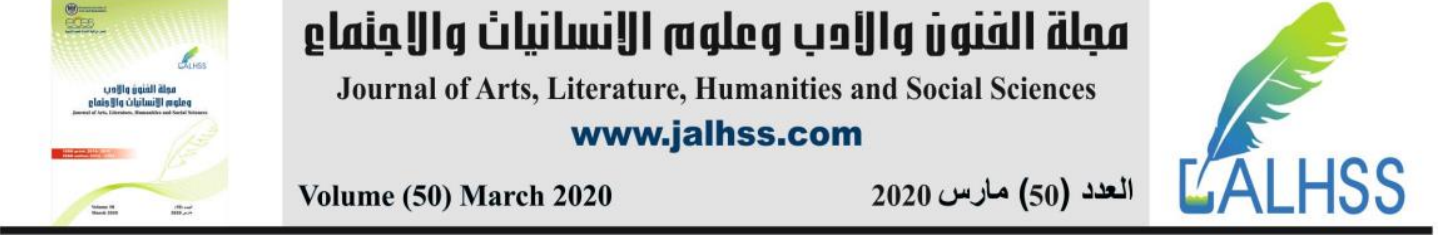

ان التورة التكنولوجية و عصر المعلومات و البيانات الضخمة الذي نعيشه منذ عقود قلبلة، أدى إلى ظهور

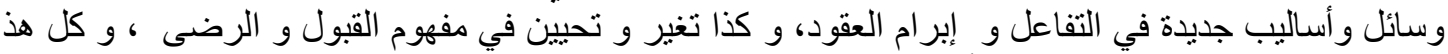

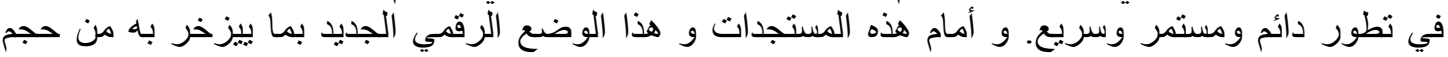

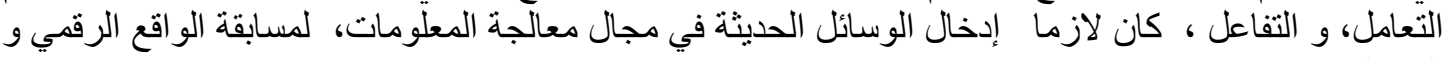
التكنولوجي.

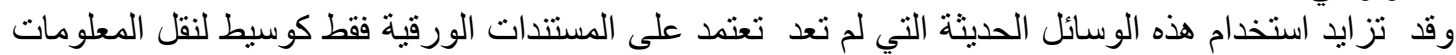

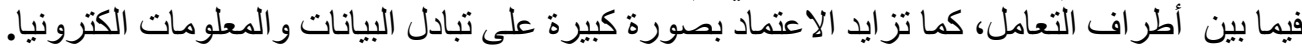

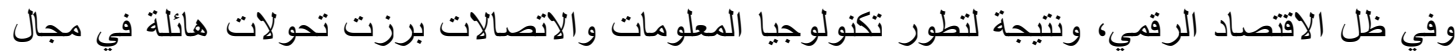

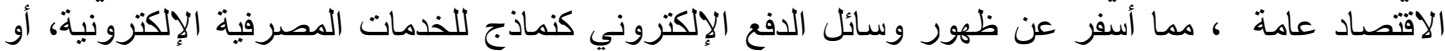

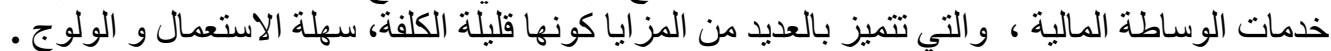

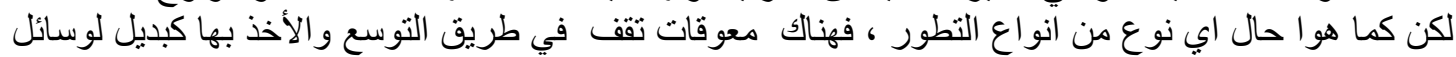

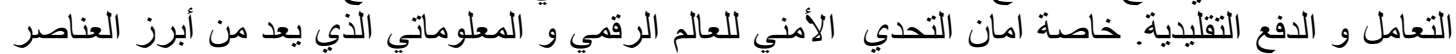

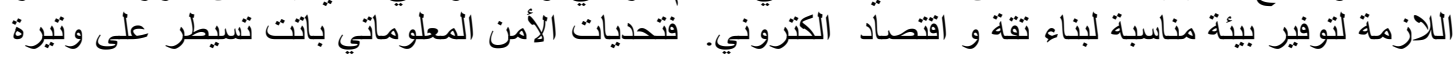
تطور الاقتصاد الرقمي بفعل تحديات الجر ائم الإكترونية وعمليات القرصنة و الاختطاف الرقمي.

\section{التوقيع الإكتروني}

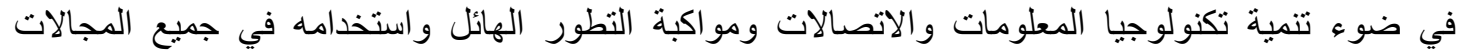

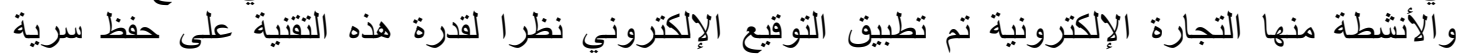

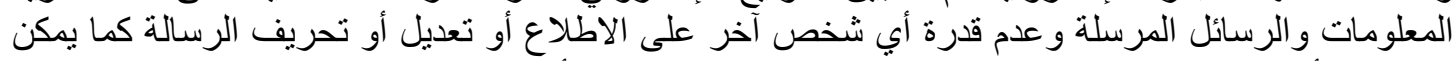

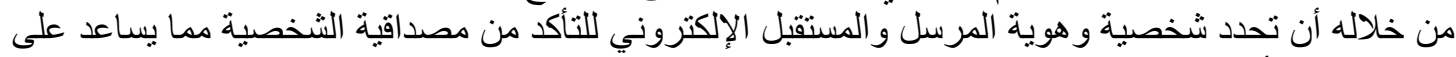

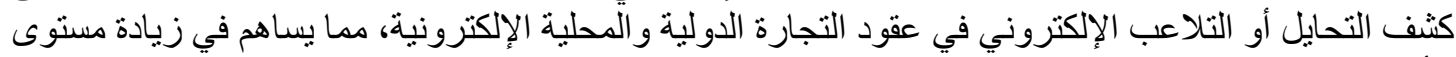

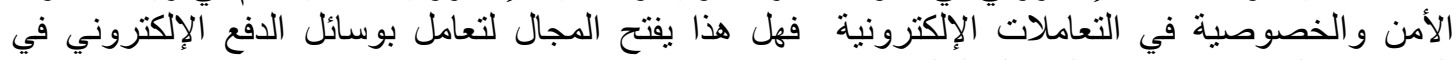
المبادلات التجارية بدلا عن الوسائل التقليدية؟. الألئ.

من خلال هذا البحت سنقوم بتسليط الضوء على مختلف التعاريف القانونية و الفقهية لمفهوم التوقيع الإلكتروني ثم نبين موقع التوقيع الإلكتروني بين الهوية و الإر ادة ومكانته كحجية قانونية ملزمة.

\section{مفهوم التوقيع الإكتروني بين الفقه القانوني و التشريعات المحلية}

وردت عدة تعريفات فقهية للتوقيع الإلكترونية حيث عرفه جانب من الفقه بأنه:” إنشارة

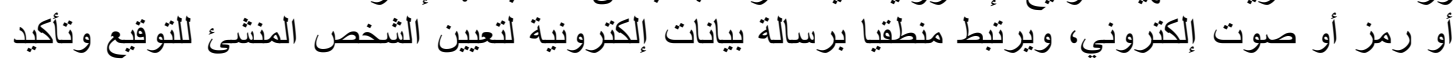

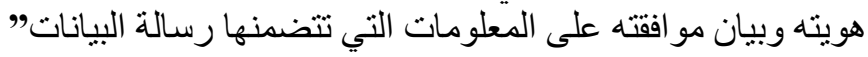

و عرفه جانب آخر من الفقه على أنه:” وحدة قصبرة من البيانات تحمل علامة رياضية مع البيانات الموجودة في

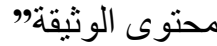

أما الجانب الثالث من الفقه فعرفه أنه:"حروف وأرقام أو رموز أو إثار ات لها طابع منفرد تشمح بتحديد شخص

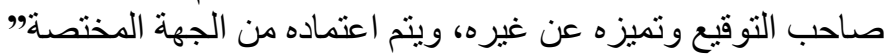

فهذه التعريفات الفقهية عرفت التوقيع الإكتروني ثم بينت الدور الوظيفي له في تعيين هوية صاحب التوقيع وتمييزه عن غيره. 


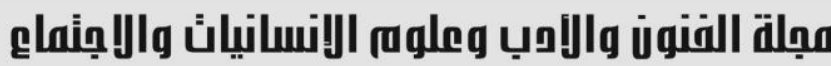

Journal of Arts, Literature, Humanities and Social Sciences

www.jalhss.com

أما التعريف التشريعي للتوقيع الإلكتروني فعرفه قانون الأوسنيتر ال النموذجي بشأن التوقيعات الإلكترونية في

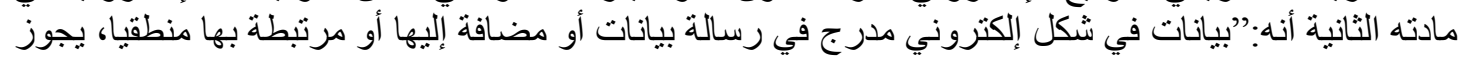

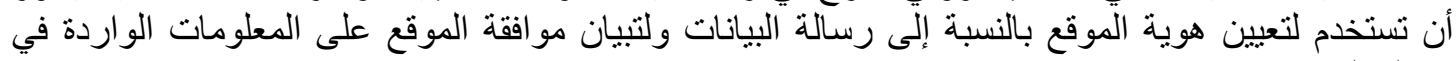

رسالة البيانات.

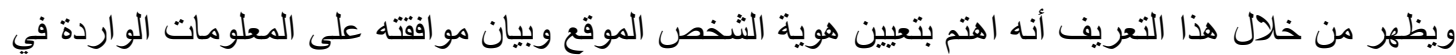

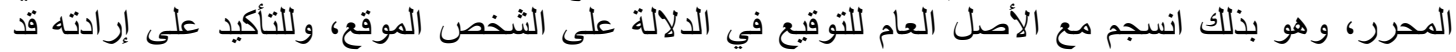

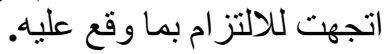

كما لم تميز بعض القوانين بين التوقيع التقليدي و التوقيع الإلكتروني من حيث الدور الوظيفي الذي يقوم به كل

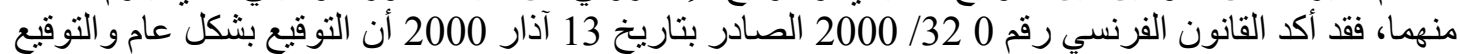

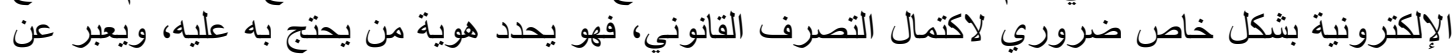

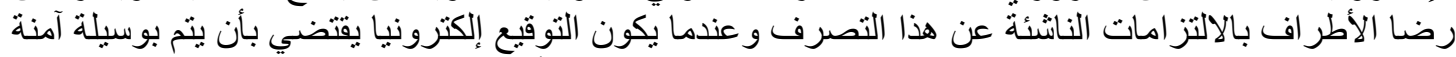

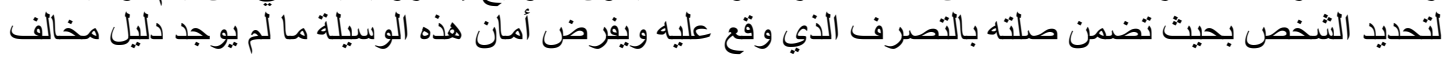

أما التشريعات العربية فقد اهتمت بالتوقيع الإلكتروني و عمل بعضها على تنظيم قوانين خاصة به كالتشريع السوري و التشريع المصري و التشريع المغربي.

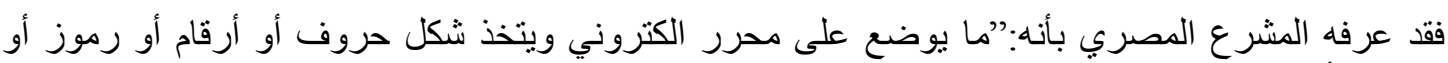

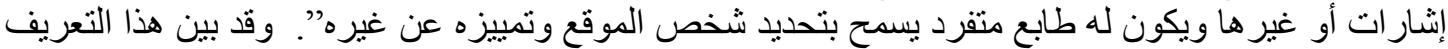

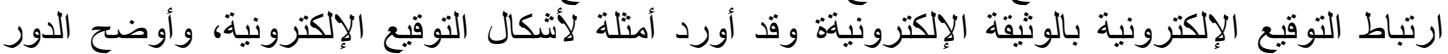
الوظيفي للتوقيع الإلكترونية في تحديد هوية المونع.

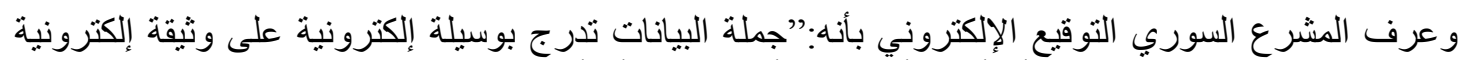

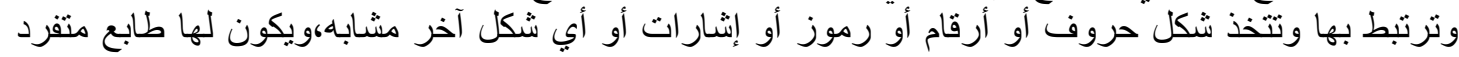

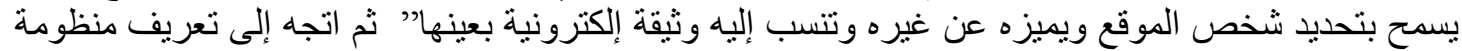

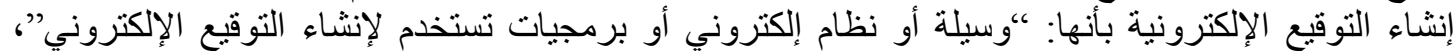

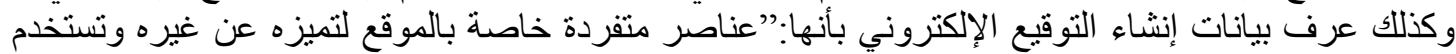
في إنشاء التوقيع الإلكتروني.ت

واعتمد المشرع السوري في تعريفه للتوقيع الإلكتروني على بيان الطبيعة الإلكترونية التي بتخذها التوقيع

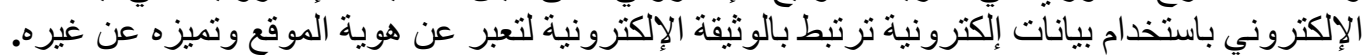

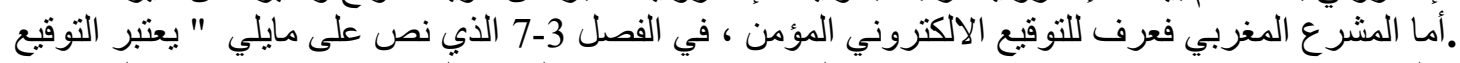

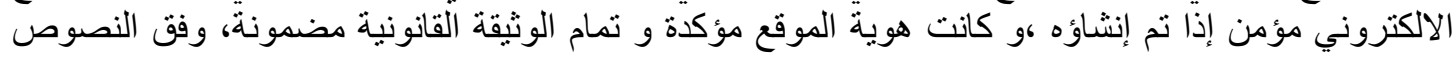

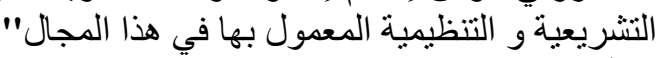

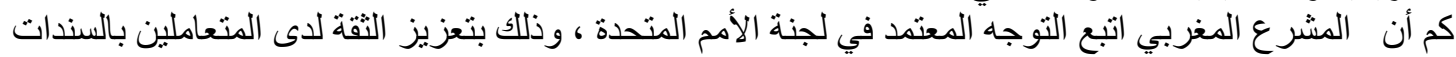

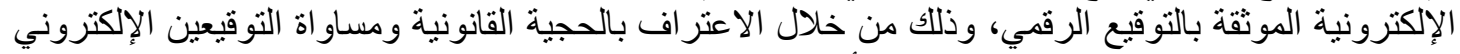

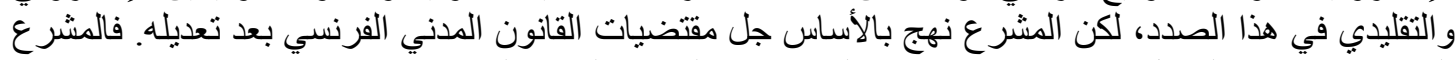

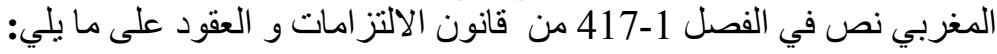

6تتمتع الوثثقة المحررة على دعامة إلكترونية بنفس قوة الإثبات التي تتمتع بها الوثيقة المحررة على الورق. تقبل الوثثقة المحررة بشكل إلكتروني للإثبات، شأنها في ذلك شأن الوثنية المحررة على الورق، شريطة أن يكون

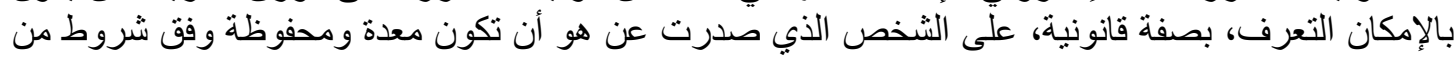




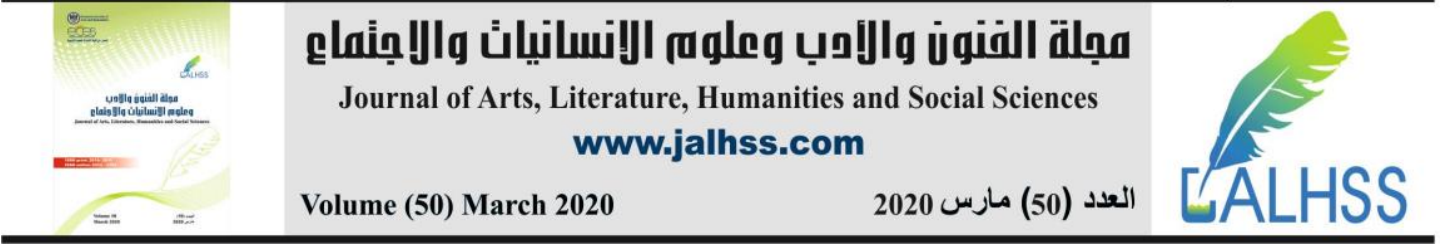

فمن خلال الفصل المذكور أعلاه سوى المشرع المغربي بين التوقيعين التقلبدي والإلكتروني في مجال الإثبات

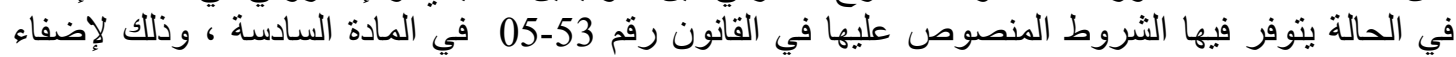
الحجية القانونية على التوقيع الرقمي،

و الفصل3-417 يؤكد على ضرورة الوثوق في التوقيع الإلكتروني المؤمن.

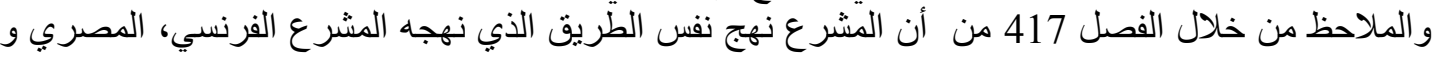

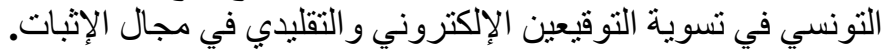

ومن ناحية أخرى لم تضع بعض التشريعات العربية قانوناً خاصاً بالتوقيع الإلكتروني إنما نظمته من خلال قانون إنبان

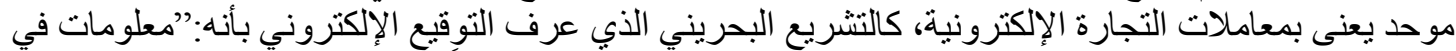

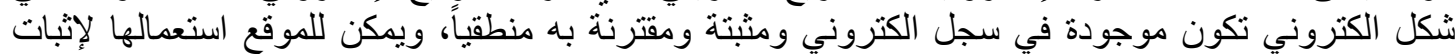

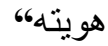

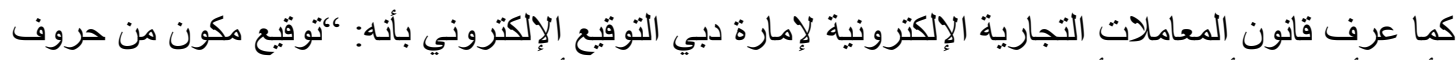

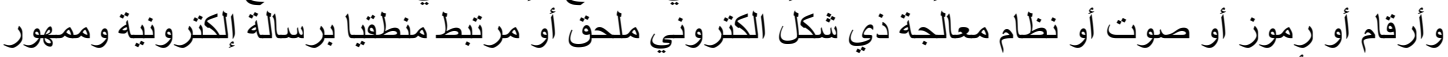

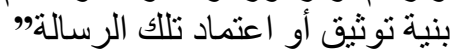

ويتضح من التعريفات السابقة الفقهية دنها والتشريعية أن التوقيع الإلكتروني وسيلة حديثة لتحقيق شرطي هما

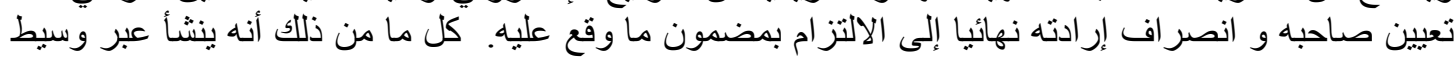
الكتروني وذللك استجابة لنوعية المعاملات التي تتم الكترونيا، فحيث تبرم العقود و الصفقات الكتر الكترونيا وجب أن أن النيط

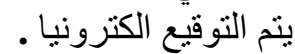

\section{التوقيع الإكتروني بين الهوية و الإرادة}

الانفتاح على السوق العالمية من خلال شبكة الانترنت سمح للمستهلكين في افتناء السلع والخدمات بأفضل

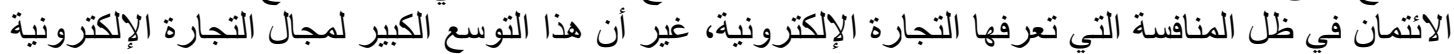

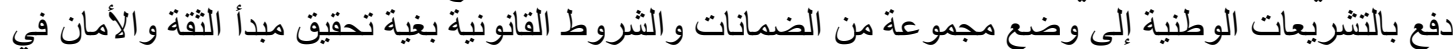

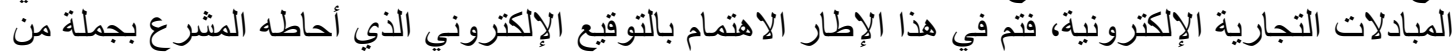
شروط الأمان، ووضع لله آلية آمنة لإنشائه حتى يكون مؤمنا وله حجية إلثبات في الإنية عمليات الدفع الإلكترونية.

\section{الهوية الرقمية والتعبير عن الإرادة}

إعطاء الهوية الرقمية لحامل وسيلة الدفع الإلكتروني: يلعب التوقيع الإلكتروني دورا كبيرا في تحديد هوية

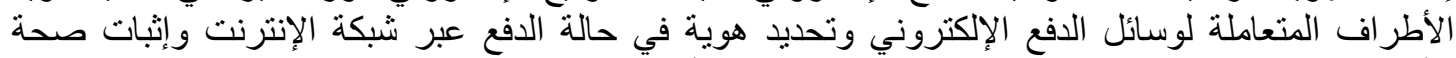

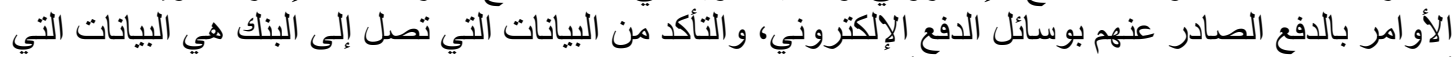

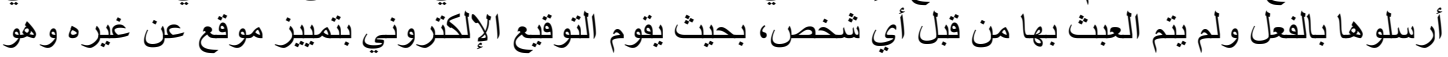

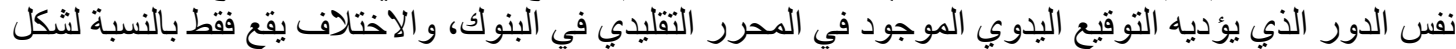

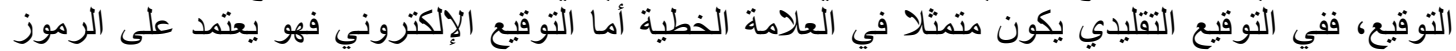

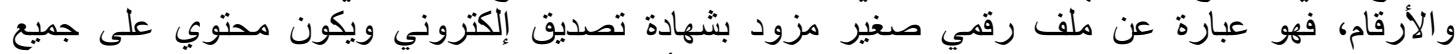

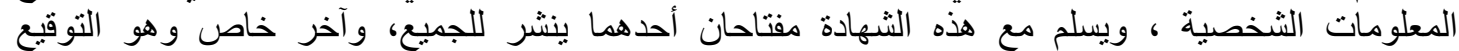

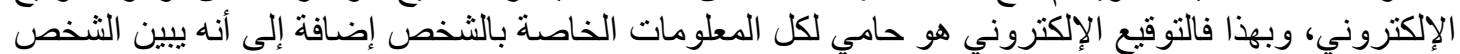

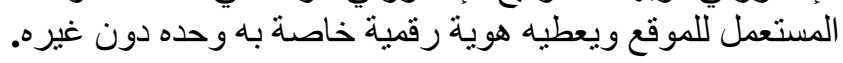




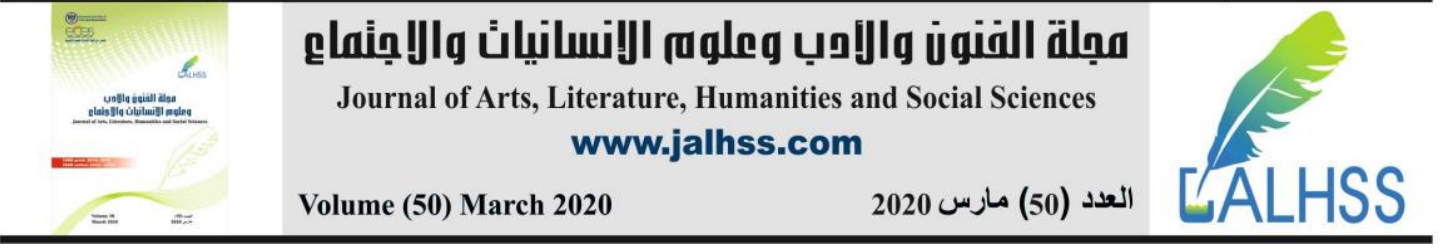

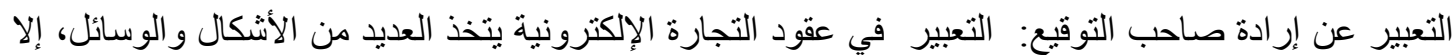

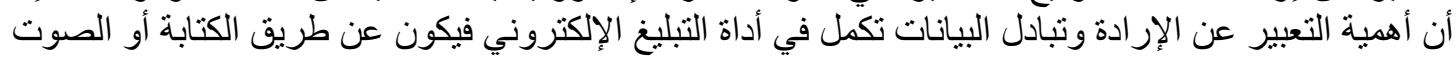

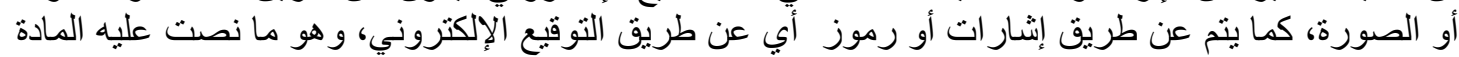

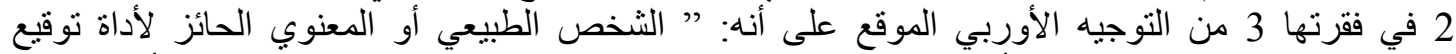

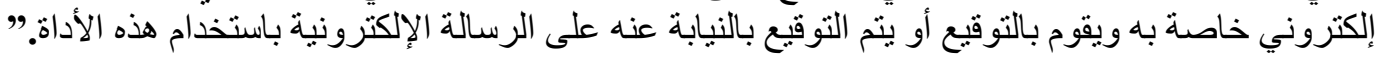

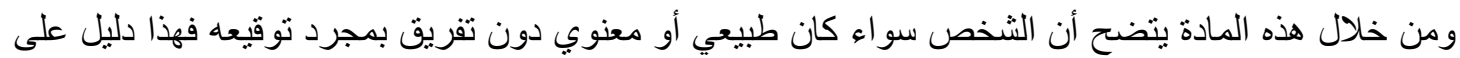

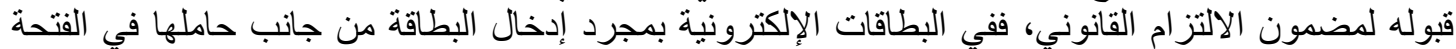

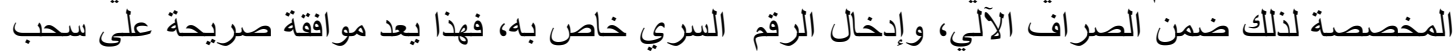
المبلغ المطلوب و المبين أمامه على شانشة الجهاز.

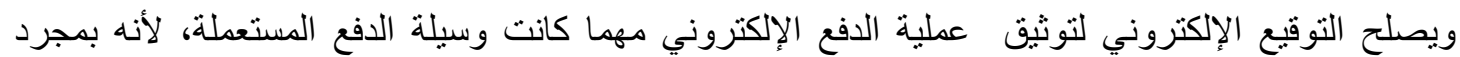

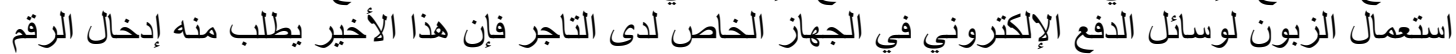

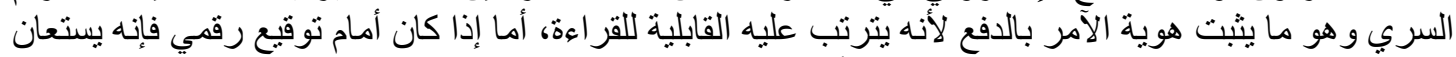

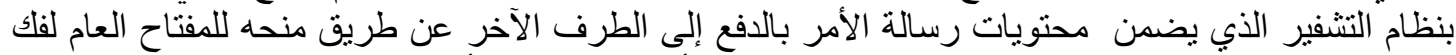

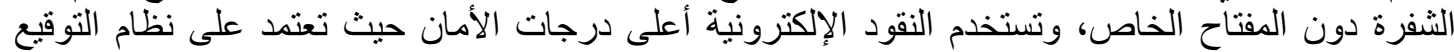

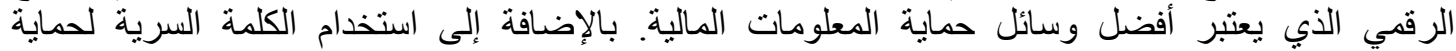
مسحوبات العميل من حسابه المصرفي، أما التوقيع البيومتري للتحقق من هوية الموقع و التي لا تكون منطابقة اللإية لدى اثنين

\section{حجية التوقيع الإكتروني في إثبات الدفع الإكتروني}

تكتسي الحجية القانونية للتوقيع الإلكتروني أهمية بالغة في الإثبات الإلكتروني، وبذانلك تحقيق حماية للمتعاملين

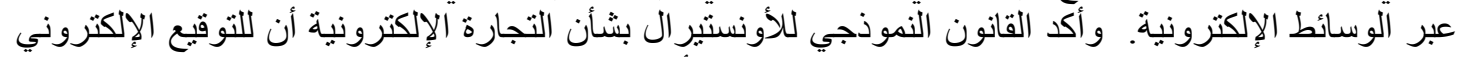

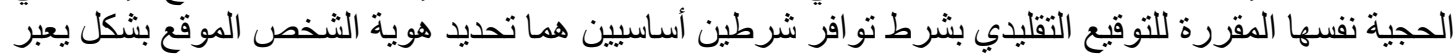

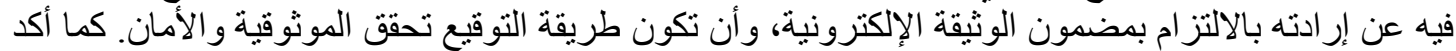

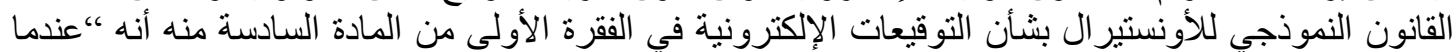

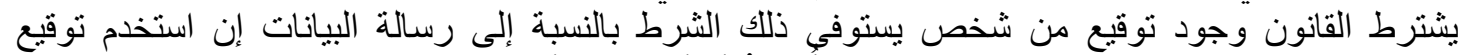

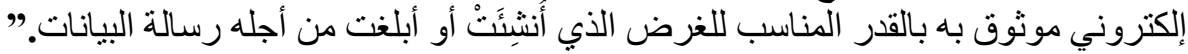

فعندما اشترط قانون الأونسيتر ال النموذجي بشأن التجارة الإلكترونية شرطين لتمتع التوقيع الإلكتروني بالحجية

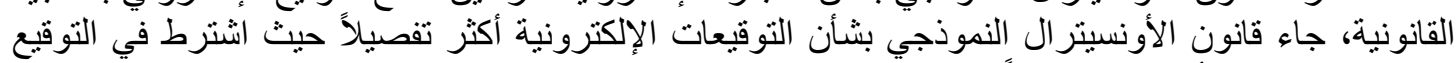

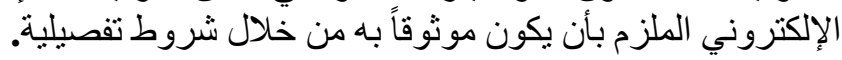

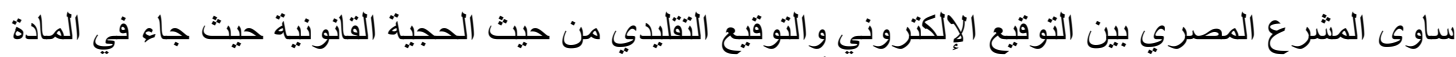

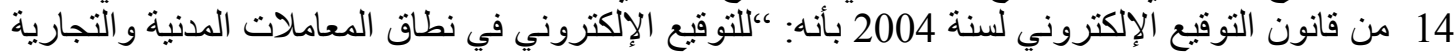

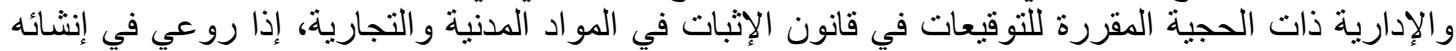

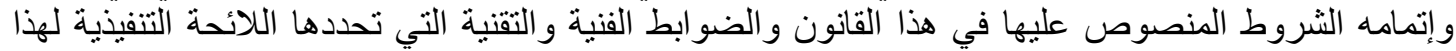

القانون.

ونفس الأمر بالنسبة إلى المشرع البحريني إذ أكد في المادة السادسة من قانون المعاملات الإلكترونية حجية التوقيع الإلكتروني فجاء فيها: 


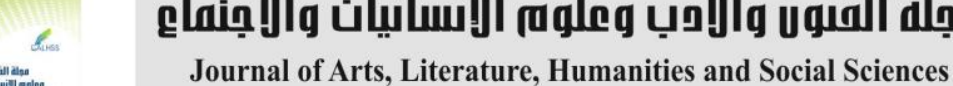
www.jalhss.com

1-1-لا ينكر الأثر القانوني للتوقيع الإكتروني من حيث صحته وإمكان العمل بموجبه لمجرد وروده كلياً أو جزئياً

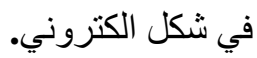

2-إذا أوجب القانون التوقيع على المستند أو رتب أثراً قانونياً على خلوه من الثوقيع فإنه إذا استعل في سجل إلكتروني في هذا الثأن فإن التوقيع الإلكتروني عليه يفي منطلبات هذا القانون.

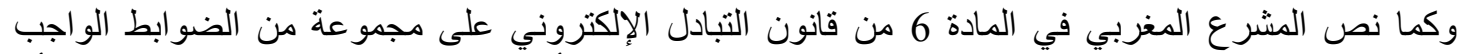

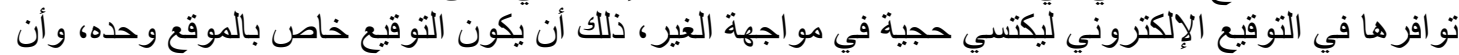

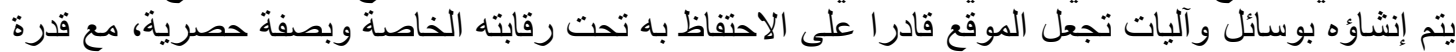
ضمان وجود ارتباط بالوثيقة المتصلة بالتوقيع الإلكتروني بطريقة تجعل طريقة الكثف أب أي تغيير لاحق أدخل

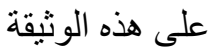

خلاصة

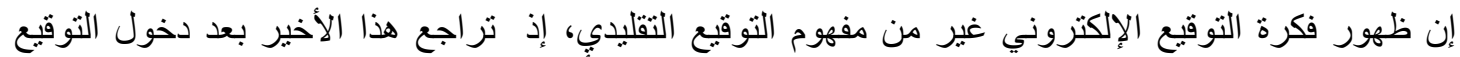

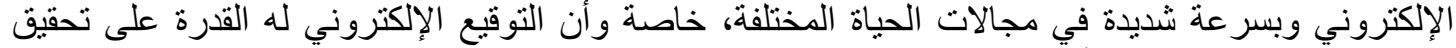
وظائف التوقيع التقليدي، بل أكثر من ذلكى، فإنه يتوفق على التوقيع التقليدي في كونه يحقق سلامة العقد.

كما يؤدي التوقيع الإلكتروني دورا هاما في تأمين مجال الدفع الإلكتروني مما يمكن أن يعتري الأجهزة والنظم

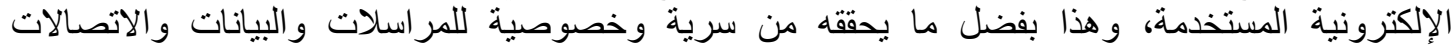

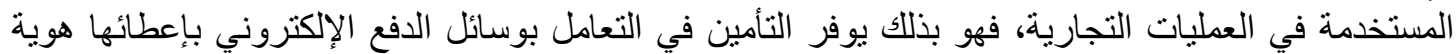
رقمية وحجية في التعامل والإثبات. وفي الاخير يمكنتا أن نبز اهم ميزات التوقيع الرقمي و هي :

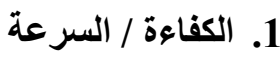

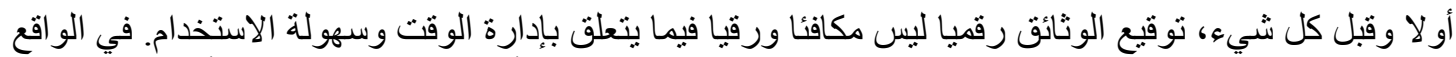

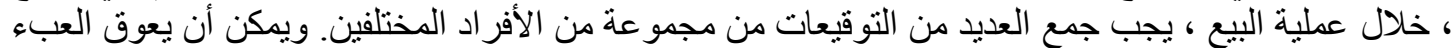

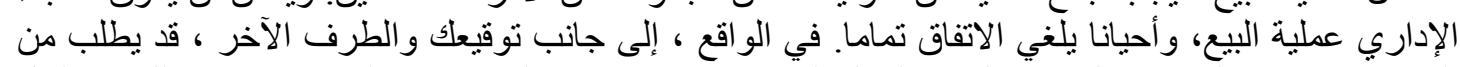

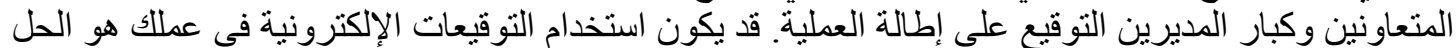
الموفر للتكاليف الذي يحدث الفرق. الوقت هو المال؛

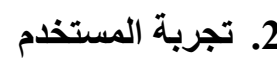

ميزة التقليل الثانية هي تعزيز تجربة المستخدم: توقيع المستندات لم يكن بهذه السهولة. عملية الطباعة و التوقيع و المسح الضوئي لإرسال نفس المستند تبسيط لفتح طلب توقيع البريد الإلكتروني. تستغرق العطلية بأكملها أقل من ليع دقيقتنن. بالإضافة إلى ذلك، واجهة المستخدم قابلة للتخصيص وشخصية مع العلامة التجارية والألوان التي تمكن من

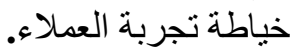

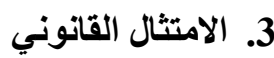

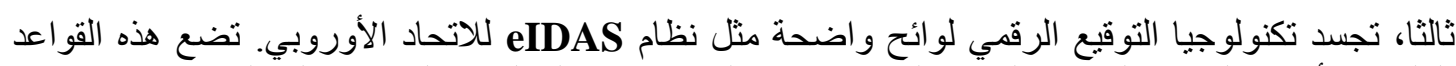

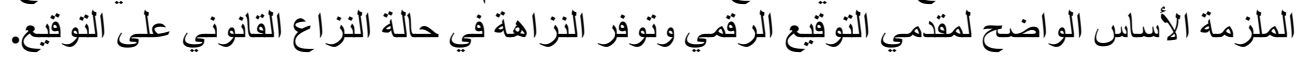




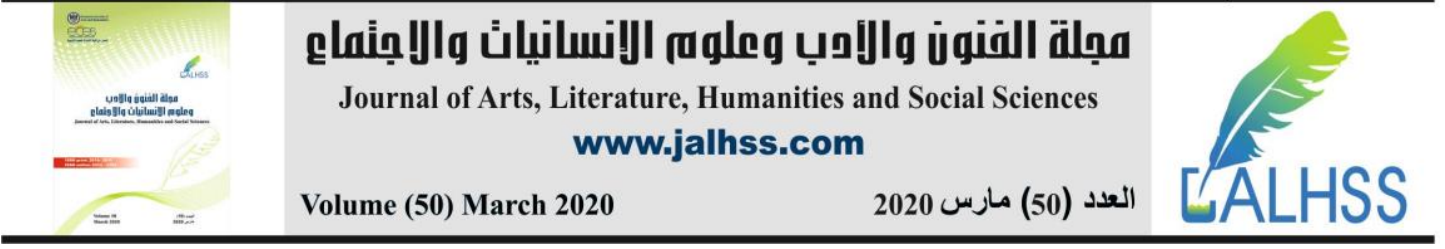

علاوة على ذلك ، تتطلب الأصالة تحديد الهوية عن طريق عاملين أو حتى عن طريق التحقق من الهوية.

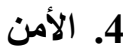

رابعا، كما ذكر للتو، هناك عاملان يوفران التحقق من الهوية والتحقق من الهوية مستوى عاليا من الأمان فيما

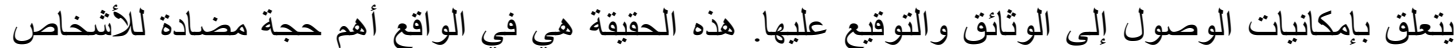

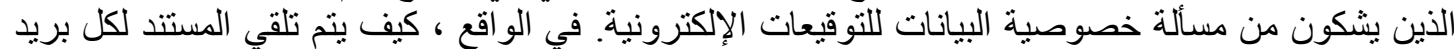

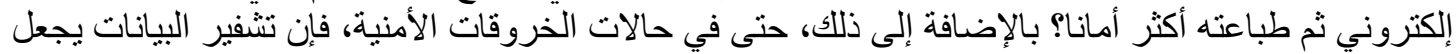

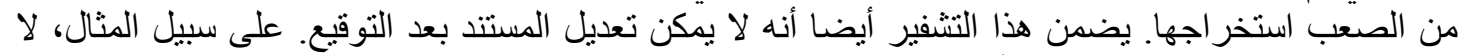
يو جد خطر من رؤية صفحة جديدة أو صفحة واحدة يتم إز التها من الاتفاقية.

إذا لم يكن هذا كافيا، فإن - 2FA وهما عاملان يمجدان - يضمنان أنه لا يمكن لأحد نسخ أو تزوير توقيعك و استخدامه في سياق آخر.

فيما يتعلق بخيار ات النشر ، يستخدم معظم موفري التو اقيع الرقمية تكوينSAAS ؛ ومع ذللك ، فإن النشر الداخلي هو اتجاه منز ايد ويمكننا أن نتوقع من جميع مقدمي الخدمات تمكين مثل هذا الخيار في المستقبل القريب.

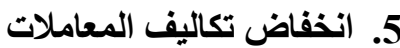

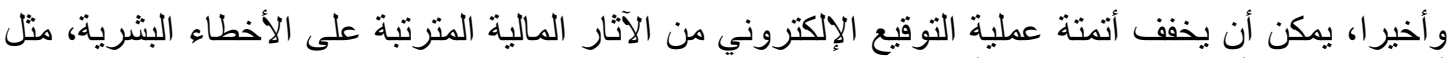

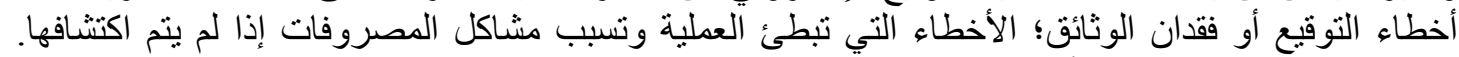

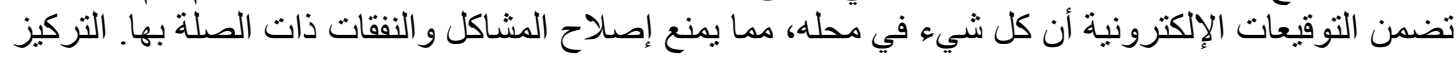

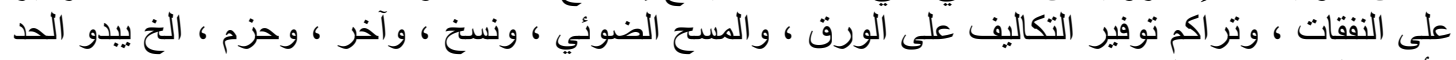
الأدنى ولكن خلاصة لتحقيق و وفور الترات كبيرة.

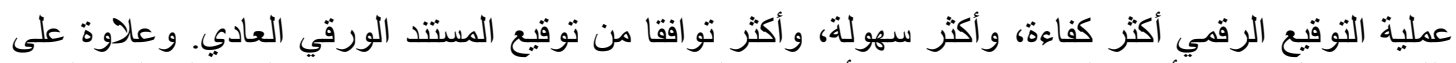

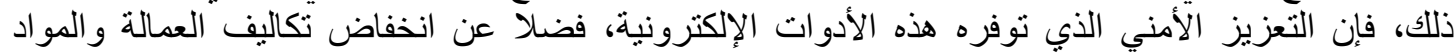
المعنية، هي مز ايا رئيسية للتوقيعات الإلكترونية الأنية

1المراجع سعبد الغربب، التوقيع الإكثروني وحجيته في الإثبات، المنشورات العربية لتنمية الإدارية، مصر،

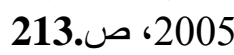

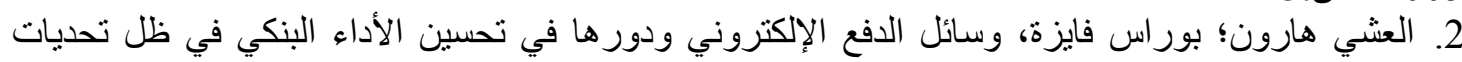

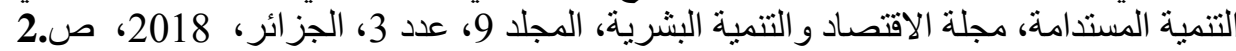

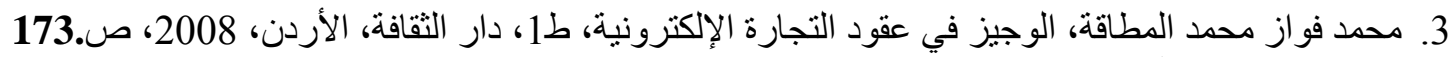
4. عادل رمضان الآيبوكي، التوقيع الإلكتروني في التشريعات الخليجية، دراسة مقارنة، المكتب الإنبة الجامعي

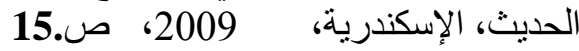
5. أبو زيد محمد محمد، تحديث في قانون الإثبات (مكانة المحررات الإلكترونية بين الأدلة الكتابية)،د.د.ن، مصر، 6. الياس ناصيف، العقد الالكتروني، العقود الدولية في القانون المقارن ، منشورات الحلبي الحقوقية، الطبعة 8. المادة 1 من قانون التوقيع الإلكتروني السوري الإنري رقم 4 بتاريخ.25/2/2009 


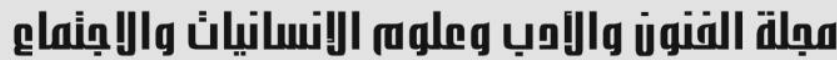

Journal of Arts, Literature, Humanities and Social Sciences
www.jalhss.com
$=2$

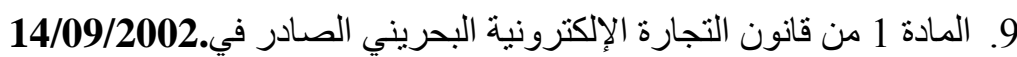

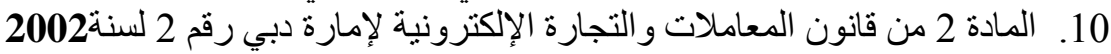

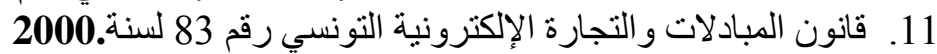

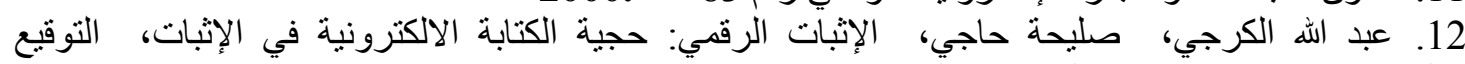

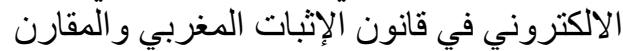

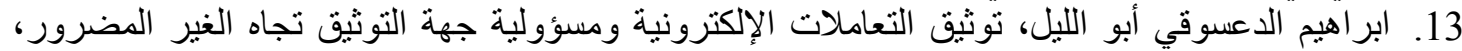

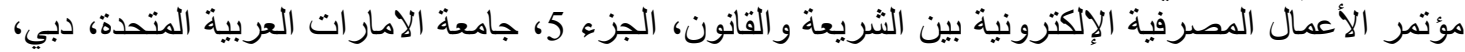

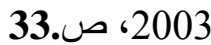

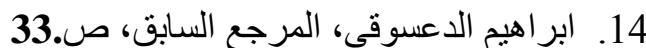
15. مصطفى كمال طه؛ وائل أنور بندق، الأوراق التجارية ووسائل الدفع الإلكترونية الحديثة، دار الفكر

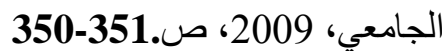

16. مجيد حميد العنبكي، سندات الثحن وتطوير أساليب النقل، مجلة العلوم، مطبعة العاني، بغداد ، 1988،

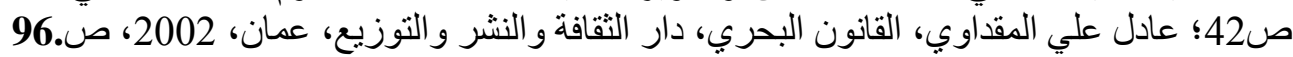
17. علاء التميمي، التنظيم القانوني للبنك الإلكتروني، على شبكة الانترنت، دارئ، دار الجامعة الجديدة للنشر ، مصر، 2012، صب641. 18. سامي عبد الباقي، الوفاء الإلكتروني بالديون الناشئة عن المعاملات التجارية، دار النهضة العربية، مصر، 110. ص 19. حو الف عبد الصمد، دور التوقيع والتصديق الإلكترونيين في تأمين وسائل الدفع الإلكتروني، مجلة كلية

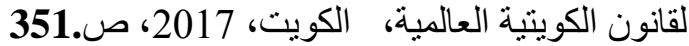
20. المصطفى طابل، أهمية التوقيع الالكتروني الآمن في حماية المتعاقدين عبر الأنترنت، مجلة جيل للأبحاث

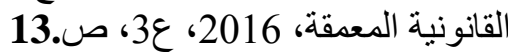

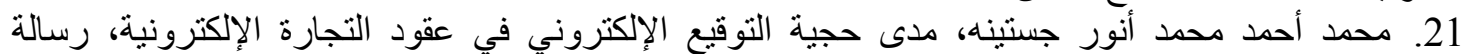

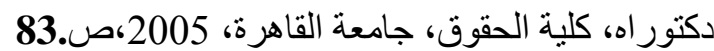
22. أحمد السيد لبيب، الدفع بالنقون، الإهدة الإلكترونية الماهية والتنظيم القانوني دراسة تحليلية مقارنة، دار الجامعة

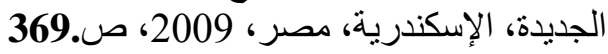

